\title{
Predicting prognosis of endometrioid endometrial adenocarcinoma on the basis of gene expression and clinical features using Random Forest
}

\author{
FUFEN YIN $^{1 *}$, XINGYANG SHAO $^{2,3^{*}}$, LIJUN ZHAO $^{1}$, XIAOPING LI $^{1}$, JINGYI ZHOU $^{1}$, \\ YUAN CHENG $^{1}$, XIANGJUN HE ${ }^{1}$, SHU LEI $^{1}$, JIANGENG LI $^{2,3}$ and JIANLIU WANG ${ }^{1}$ \\ ${ }^{1}$ Department of Obstetrics and Gynecology, Peking University People's Hospital, Beijing 100044; \\ ${ }^{2}$ College of Automation, Faculty of Information Technology, Beijing University of Technology; \\ ${ }^{3}$ Beijing Key Laboratory of Computational Intelligence and Intelligent System, Beijing 100124, P.R. China
}

Received September 6, 2018; Accepted April 3, 2019

DOI: $10.3892 / \mathrm{ol} .2019 .10504$

\begin{abstract}
Traditional clinical features are not sufficient to accurately judge the prognosis of endometrioid endometrial adenocarcinoma (EEA). Molecular biological characteristics and traditional clinical features are particularly important in the prognosis of EEA. The aim of the present study was to establish a predictive model that considers genes and clinical features for the prognosis of EEA. The clinical and RNA sequencing expression data of EEA were derived from samples from The Cancer Genome Atlas (TCGA) and Peking University People's Hospital (PKUPH; Beijing, China). Samples from TCGA were used as the training set, and samples from the PKUPH were used as the testing set. Variable selection using Random Forests (VSURF) was used to select the genes and clinical features on the basis of TCGA samples. The RF classification method was used to establish the prediction model. Kaplan-Meier curves were tested with the log-rank test. The results from this study demonstrated that on the basis of
\end{abstract}

Correspondence to: Professor Jianliu Wang, Department of Obstetrics and Gynecology, Peking University People's Hospital, 11 Xizhimen South Street, Xicheng, Beijing 100044, P.R. China

E-mail: wangjianliu@pkuph.edu.cn

Professor Jiangeng Li, College of Automation, Faculty of Information Technology, Beijing University of Technology, 100 Ping Le Yuan, Chaoyang, Beijing 100124, P.R. China

E-mail: lijg@bjut.edu.cn

${ }^{*}$ Contributed equally

Abbreviations: EEA, endometrioid endometrial adenocarcinoma; TCGA, The Cancer Genome Atlas; PKUPH, Peking University People's Hospital; VSURF, variable selection method using Random Forests; PE, primary EEA; R/D-PE, relapsed or deceased primary EEA; RF, Random Forest

Key words: endometrioid endometrial adenocarcinoma, prognostic, model, Random Forest, feature selection
TCGA samples, 11 genes and the grade were selected as the input features. In the training set, the out-of-bag (OOB) error of RF model-1, which was established using the '11 genes', was 0.15 ; the OOB error of RF model-2, which was established using the 'grade', was 0.39; and the OOB error of RF model-3, established using the ' 11 genes and grade', was 0.15 . In the testing set, the classification accuracy of RF model-1, model-2 and model-3 was $71.43,66.67$ and $80.95 \%$, respectively. In conclusion, to the best of our knowledge, the VSURF was used to select features relevant to EEA prognosis, and an EEA predictive model combining genes and traditional features was established for the first time in the present study. The prediction accuracy of the RF model on the basis of the 11 genes and grade was markedly higher than that of the RF models established by either the 11 genes or grade alone.

\section{Introduction}

Among the different forms of endometrial cancer, endometrioid endometrial adenocarcinoma (EEA) is the most common type (85\%) (1). Although the prognosis of EEA is good, extensive heterogeneity has been reported in a number of studies, particularly in patients with an early stage of disease, exposing women to recurrent disease (2-4). Clinically, certain patients with EEA with an advanced stage of disease have a good prognosis, whereas certain patients with an early stage of disease can still relapse and succumb $(5,6)$. All these features indicate that traditional clinical features are not sufficient to accurately predict the prognosis of EEA. Molecular biological characteristics and traditional clinical features are particularly important in the prognosis of EEA. Tumor occurrence and development are driven by genetic alterations, and the phenotypic diversity may be accompanied by the corresponding diversity in the pattern of gene expression (7). Therefore, establishing a predictive prognostic model on the basis of gene expression profiles and traditional clinical features, which are different from the traditional criteria, is of great clinical value.

Machine-learning methods have been used to predict the prognosis of numerous types of cancer $(8,9)$. In the machine-learning area of research, the prognosis of cancer is a 
typicalclassification problem. When training a machine-learning model to undergo a prediction task, the factors relevant to the prognosis of cancer can be regarded as the features of the data, and the prognosis results are the class labels. Random Forest (RF) is a type of machine-learning method, which has been experimentally proven to be the best classifier (10). RF has a number of advantages and has already been successfully applied to microarray data classification $(11,12)$ and numerous other disease classifications $(13,14)$. Among the different variable selection methods, variable selection using RF (VSURF) has demonstrated the best predictive performance thus far (15). VSURF can handle thousands of input variables and identify the most significant variables (10); thus, it is considered a feature selection method and has been used to select the genes relevant to the type of cancer in question $(11,16)$.

However, to the best of our knowledge, there is currently no RF for predicting EEA prognosis by combining gene expression and traditional clinical characteristics. Therefore, the aim of the present study was to establish a prediction model combining genes and clinical features via RF for the prognosis of EEA. First, the state-of-the-art method VSURF was used to select informative factors that are relevant to the prognosis of EEA. The selected factors were then used to design an accurate predictive model via RF.

\section{Materials and methods}

Patient selection. The present study was performed in the Department of Obstetrics and Gynecology, Peking University People's Hospital (PKUPH; Beijing, China). In the training cohort, 154 primary EEA (PE) samples without neoadjuvant therapy, RNA-sequencing (RNAseq) expression (combining level 3 data) and clinical data of female patients with uterine cancer were obtained from The Cancer Genome Atlas (TCGA) data portal (cancergenome.nih.gov) on January 7, 2018. These data included $64 \mathrm{PE}$ samples without relapse ( $\geq 3$ years of clinical follow-up), without radiation therapy and without additional pharmaceutical treatment, and 90 samples from relapsed or deceased PE (R/D-PE) patients with or without postoperative adjuvant therapy. TCGA samples were sub-stratified into four molecular subgroups: i) Copy number low (CN-L), ii) copy number high (CN-H), iii) microsatellite instability (MSI) and iv) catalytic subunit of DNA polymerase $\varepsilon$ involved in nuclear DNA replication and repair (POLE) ultra-mutated, with different prognoses. Of the 154 cases in TCGA training cohort, $20.13 \%$ were CN-L, $5.19 \%$ were CN-H, 33.12\% were MSI and $5.84 \%$ were POLE ultra-mutated; in $35.72 \%$ of the cases, the molecular typing information was lacking. The detailed inclusion or exclusion criteria and information on the selection of these 154 TCGA participants are presented in Fig. 1. In the testing cohort, 21 PE samples without neoadjuvant therapy, as well as RNAseq expression and clinical data, were obtained from 21 surgically treated patients at the Department of Obstetrics and Gynecology PKUPH. All 21 samples were from patients without neoadjuvant therapy and who underwent surgical resection between January 2008 and December 2012. The cohort included $13 \mathrm{PE}$ samples from patients without relapse ( $\geq 3$ years of clinical follow-up) and 8 R/D-PE samples from patients with or without adjuvant therapy. The EEA samples were divided into two groups according to the prognosis. The group with a good prognosis contained the samples from non-relapsed EEA patients, and the group with a poor prognosis contained the samples form relapsed or deceased EEA patients. All deceased patients had succumbed to EEA. The study was approved by the Institutional Ethics Committee (Human Research) of the PKUPH.

RNA isolation, RNAseq library preparation and sequencing of the 21 EEA samples. The total RNA was extracted with TRIzol $^{\circledR}$ (Tiangen Biotech Co., Ltd., Beijing, China) and assessed with an Agilent 2100 BioAnalyzer instrument (Agilent Technologies, Inc., Santa Clara, CA, USA) and a Qubit $^{\mathrm{TM}} 4$ Fluorometer (Invitrogen; Thermo Fisher Scientific, Inc., Waltham, MA, USA). The total RNA samples that met the following requirements were used in subsequent experiments: RNA integrity number $>7.0$ and a $28 \mathrm{~S} / 18 \mathrm{~S}$ ratio $>1.8$. RNAseq libraries were generated and sequenced by CapitalBio Technology Co., Ltd. (Beijing, China). Triplicate samples of all assays were constructed in an independent library. The NEB Next Ultra RNA Library Prep kit for Illumina (New England BioLabs, Inc., Ipswich, MA, USA) was used to construct the DNA libraries for sequencing. The NEB Next Poly(A) mRNA Magnetic Isolation Module kit (New England BioLabs, Inc.) was used to enrich the poly(A)-tailed mRNA molecules from $1 \mu \mathrm{g}$ total RNA. The mRNA was fragmented into 200 -bp pieces. The first-strand cDNA was synthesized from the mRNA fragments using reverse transcriptase and random hexamer primers (New England BioLabs, Inc.), and the second-strand cDNA was synthesized using DNA polymerase I and RNaseH (New England BioLabs, Inc.). The end of the cDNA fragment was subjected to an end repair process that included the addition of a single ' $A$ ' base, followed by ligation of the adapters, according to the instructions of the NEB Next Ultra RNA Library Prep kit (New England BioLabs, Inc.). The end Repair/dA-tail program was: i) $20^{\circ} \mathrm{C}$ for $30 \mathrm{~min}$; ii) $65^{\circ} \mathrm{C}$ for $30 \mathrm{~min}$; iii) Hold at $4^{\circ} \mathrm{C}$. The products were purified using Agencourt AMPure XP Beads (Beckman Coulter, Inc., Brea, CA, USA) according to the manufacturer's protocol and enriched by polymerase chain reaction (PCR) to amplify the library DNA. Universal Primer Mix (New England BioLabs, Inc.) was used for amplification. The thermocycling conditions were as follows: $98^{\circ} \mathrm{C}$ for $30 \mathrm{sec} ; 12$ cycles of $98^{\circ} \mathrm{C}$ for $10 \mathrm{sec}, 65^{\circ} \mathrm{C}$ for $30 \mathrm{sec}$ and $72^{\circ} \mathrm{C}$ for $30 \mathrm{sec} ; 72^{\circ} \mathrm{C}$ for $5 \mathrm{~min}$. The final libraries were quantified using the KAPA Library Quantification kit (KAPA Biosystems; Roche Diagnostics, Basel, Switzerland) and an Agilent 2100 Bioanalyzer (Agilent Technologies, Inc.). The libraries were validated using reverse transcription-quantitative PCR, and the thermocycling conditions were as follows: $95^{\circ} \mathrm{C}$ for $5 \mathrm{~min}$; 40 cycles of $95^{\circ} \mathrm{C}$ for $30 \mathrm{sec}$ and $60^{\circ} \mathrm{C}$ for $45 \mathrm{sec}$. The libraries were subjected to paired-end sequencing with a pair-end 150-bp reading length on an Illumina HiSeq sequencer (Illumina, Inc., San Diego, CA, USA) (17).

Data processing. In total, 18,669 coding genes were included in TCGA RNAseq data. Fragments per kilobase of exon model per million mapped fragments (FPKM) gene expression values were used for the statistical analysis. The format of RNAseq data downloaded from the TCGA was $\log _{2}($ FPKM+1); thus, the RNAseq data of the TCGA into FPKM was transformed for 


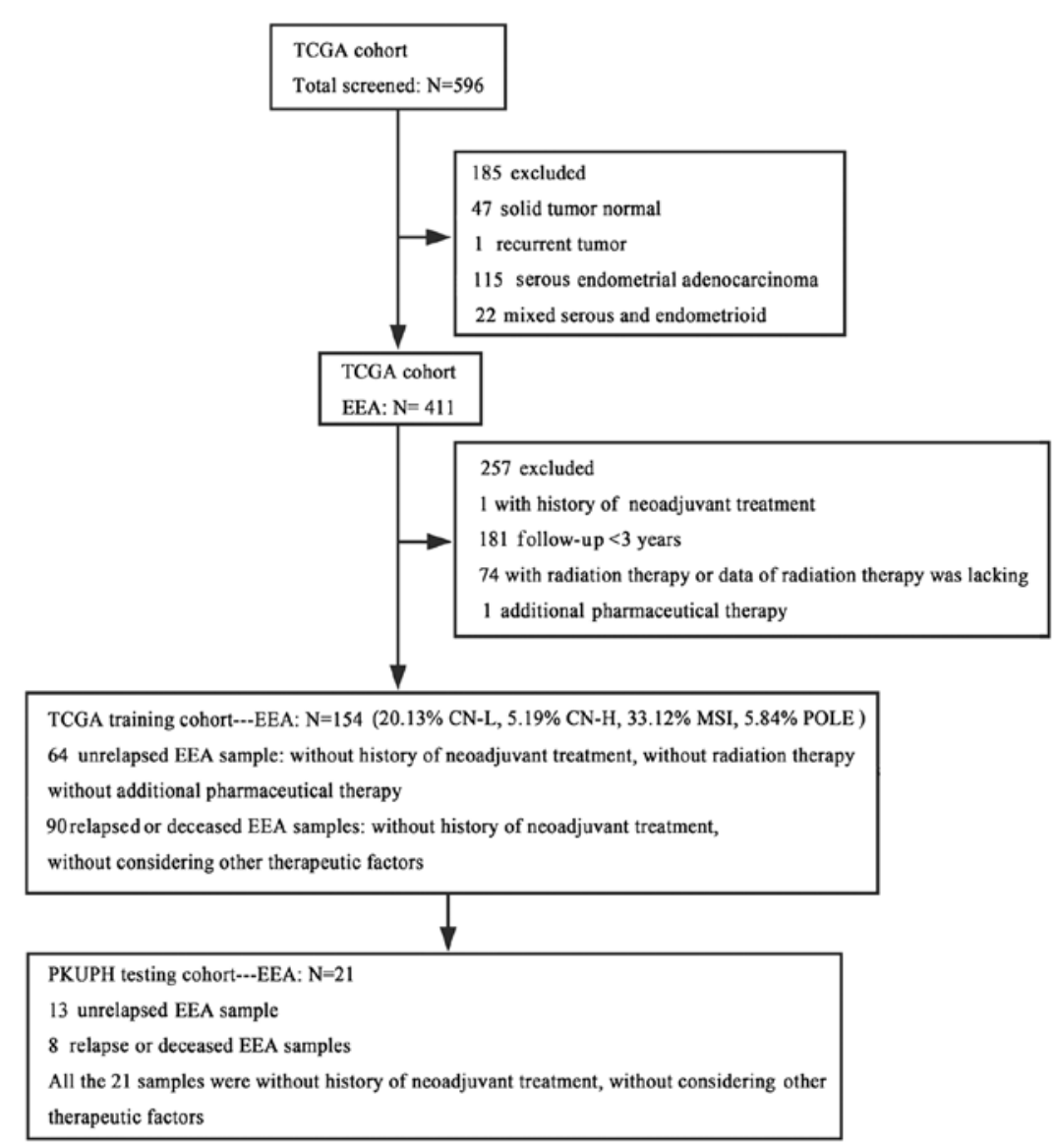

Figure 1. Flow chart of study participants. TCGA, The Cancer Genome Atlas; EEA, endometrioid endometrial adenocarcinoma; CN-L, copy number low; $\mathrm{CN}-\mathrm{H}$, copy number high; MSI, microsatellite instability; POLE, catalytic subunit of DNA polymerase $\varepsilon$ involved in nuclear DNA replication and repair, ultra-mutated; PKUPH, Peking University People's Hospital.

the follow-up study. There were a number of clinical features in TCGA clinical data, including age at initial pathological diagnosis (age), International Federation of Gynecology and Obstetrics (FIGO) stage (18), grade (19), peritoneal wash status and lymph node status. However, only the data for age, FIGO stage and grade were complete in TCGA cohorts. Thus, of all the clinical features, only age, FIGO stage and grade were included in the present study. To improve the generalization of the study results, a numerical value was given to age, FIGO stage and grade, according to a prior published study (20) and clinical experience. The numerical values of age, FIGO stage and grade were as follows: Age ( $<60$ years, 1; and $\geq 60$ years, 2.55 ), grade (I-II, 1 ; and III, 2.43), and FIGO stage (Ia, 1; Ib, 1.5; II, 2.75; IIIa-b, 4; IIIc1, 4.21; IIIc2, 4.5; and IV, 6). These numerical values were used for the establishment of the RF prognostic prediction model of EEA.

$R F$. RF is an ensemble of decision trees, which forms multiple decision trees and then aggregates them to provide a final prediction. When a new object from an input vector is to be predicted, the input vector is placed on each of the trees in the forest simultaneously. Each tree gives a prediction, then, the forest chooses the classification that has the most votes (out of all the trees in the forest). RF uses the bagging technique and the random feature selection technique. RF has two parameters, which are the number of trees (ntree) and the number of variables randomly sampled as candidates at each split (mtry).
VSURF. VSURF is a three-step feature selection method based on RF. The first step is dedicated to removing irrelevant features from the dataset. The second step aims to select important features relevant to the class labels for interpretation purposes. The third step refines the selection by removing redundancy in the set of features selected by the second step, for prediction purposes. The ntree parameter was set to its default value of 2,000, and the mtry parameter was set to its default value (if nvm, the number of variables in the model is not greater than the number of observations; otherwise it is set to nvm/3). The VSURF results of the genes and clinical features are summarized in Fig. 2, with Fig. 2Aa-b, and 2Ba-b corresponding to the 'thresholding step', Fig. 2Ac and 2Bc corresponding to the 'interpretation step', and Fig. 2Ad and $2 \mathrm{Bd}$ corresponding to the 'prediction step'. The features from the 'interpretation step' had a strong association with EEA prognosis and were determined as the most important factors that affect the prognosis of EEA.

Prediction experiment. RF parameter setting. The ntree parameter was set to 2,000 , i.e., the RF included 2,000 decision trees, and the mtry parameter was set to its default value.

Statistical analysis. All the model-associated data analyses were performed using R software (version 3.2.4; https://www.r-project.org). The VSURF method was used to select the most relevant prognostic genes and clinical 
$\mathbf{A}$

Gene selection procedure
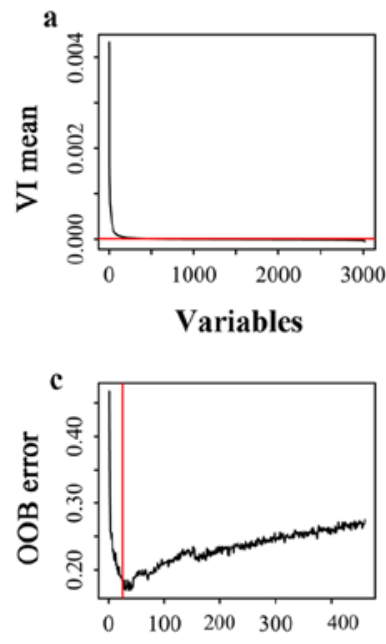

Nested models
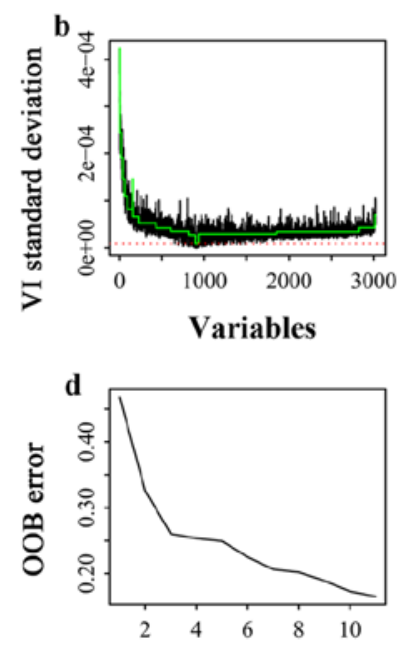

Predictive models
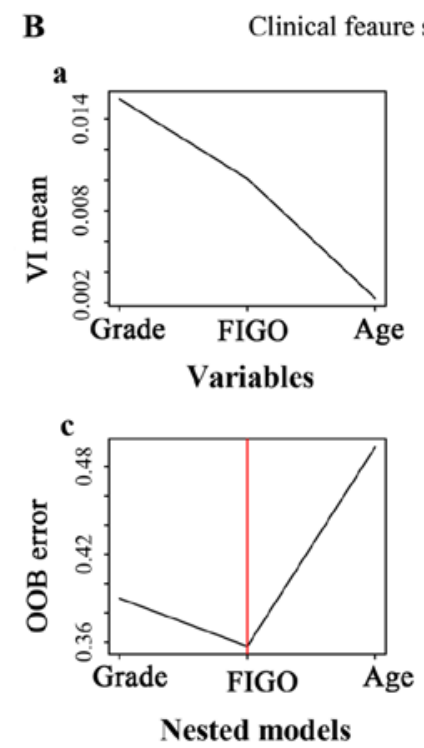
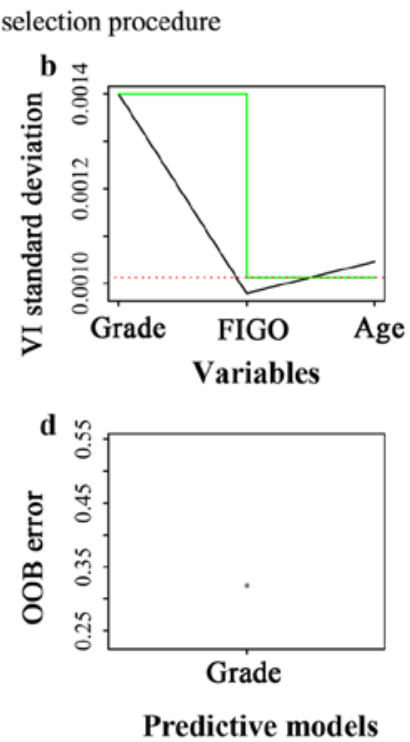

Figure 2. Feature selection procedures for the interpretation and prediction of the prognosis of EEA. (A) Gene selection procedure for the interpretation and prediction of the prognosis of EEA. (B) Clinical features selection procedure for the interpretation and prediction of the prognosis of EEA. Green and red lines are auxiliary lines used in the feature selection process. EEA, endometrioid endometrial adenocarcinoma; VI, variable importance; OOB, out-of-bag; FIGO, International Federation of Gynecology and Obstetrics.

characteristics. RF was used to build the predictive model for separating (relapsed or deceased) and unrelapsed patients. SPSS software (version 13.0; SPSS Inc., Chicago, IL, USA) was used to perform the statistical analysis. The associations between clinicopathological characteristics and outcomes were calculated using the $\chi^{2}$ test and Fisher's exact test. Survival curves for the 154 PE samples from TCGA cohort (Fig. 3) were plotted using the Kaplan-Meier method and the differences between survival curves were calculated using a log-rank test. $\mathrm{P}<0.05$ was considered to indicate a statistically significant difference.

\section{Results}

Patient characteristics. In total, $154 \mathrm{PE}$ samples meeting the inclusion criteria from TCGA cohort were selected for the training set, and $21 \mathrm{PE}$ samples from the PKUPH cohort were included in the testing set. The median age of diagnosis for the samples in TCGA cohort was 64 years (range, 35-90 years). No significant difference was observed in the diagnostic age and menopause status between the PE samples and the R/D-PE samples, whereas significant differences existed in the grade, FIGO stage, lymph node status, adjuvant radiotherapy and body mass index. The median age of diagnosis for the samples in the PKUPH cohort was 55 years (range, 31-75 years). There was no significant difference observed in all the stated clinical characteristics, perhaps due to the limited sample size in the PKUPH cohort. The detailed data are presented in Table I.

Establishing an RF prediction model on the basis of the selected genes. The VSURF method was used to select genes from 18,669 coding genes of TCGA RNAseq data for the establishment of RF prediction models, and ultimately, 11 genes were selected (Fig. 2Ab). First, 19 genes that had the most relevance to the prognosis of EEA were selected (Fig. 2Ac). To further reduce the number of genes for the RF models, 11 genes (Table II) were selected from these 19 genes as the input factors. For seven of the 11 genes [low density lipoprotein receptor class A domain-containing 2 (LDLRAD2) (OS, $\mathrm{P}<0.05$; RFS, P>0.05), 24-dehydrocholesterol reductase (DHCR24) (OS, $\mathrm{P}<0.05$; RFS, $\mathrm{P}<0.05)$, EF-hand calcium-binding domain-containing protein 6 (EFCAB6) (OS, P<0.05; RFS, $\mathrm{P}<0.05)$, epithelial-splicing-regulatory-protein 1 (ESRP1) (OS, $\mathrm{P}<0.05$; RFS, $\mathrm{P}<0.05$ ), apolipoprotein L2 (APOL2) (OS, $\mathrm{P}>0.05$; RFS, $\mathrm{P}<0.05)$, derlin-1 (DERL1) (OS, $\mathrm{P}<0.05$; RFS, $\mathrm{P}<0.05)$ and mediator complex subunit 8 (MED8) (OS, P<0.05; RFS, $\mathrm{P}<0.05)$, the gene expression was significantly associated with the survival of EEA ( $\mathrm{P}<0.05$; Fig. 3). The classification ability of the 11 genes (Fig. 2Ad) was approximately equal to the 19 genes (Fig. 2Ac). In the training set, the out-of-bag (OOB) error of RF model-1 established by the 11 genes was $15 \%$ (Fig. 2Ad). In the testing set, when RF model-1 was used to validate the 21 EEA samples from the PKUPH cohort, its classification accuracy was $71.43 \%$.

Establishing an RF prediction model on the basis of the clinical features. The VSURF method was used to select clinical features for establishing RF prediction models, and the grade was selected (Fig. 2B). The results indicated that grade and FIGO stage were the most relevant to the EEA prognosis (Fig. 2Bc). To further reduce the number of clinical factors in the RF models, grade was finally chosen as the input factor (Fig. 2Bd). Grade had an almost equal ability to assign a classification compared with the 'grade combined with FIGO stage' (Fig. 2Bc, 2Bd). In the training set, the OOB error of the RF model-2 established by grade was 0.39 (Fig. 2Bd). When RF model-2 was used to validate the 21 EEA samples from the PKUPH cohort, the classification accuracy was $66.67 \%$ (Fig. 4A).

Establishing a RF combined model on the basis of the 'genes and clinical features'. Molecular biological characteristics 


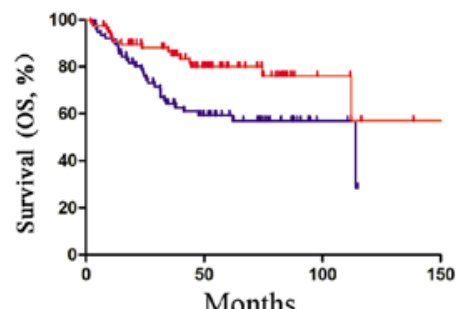

+ LDLRAD2 expression < Median + LDLRAD2 expression > Median Log-rank $P=0.0077$

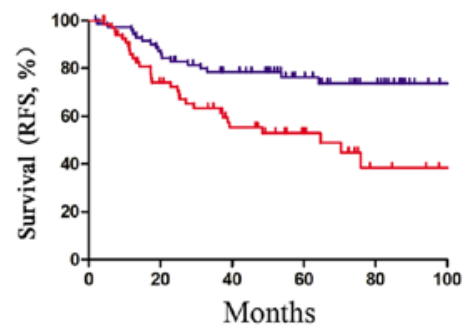

- DHCR24 expression < Median

- DHCR24 expression $>$ Median Log-rank $\mathrm{P}=0.0017$

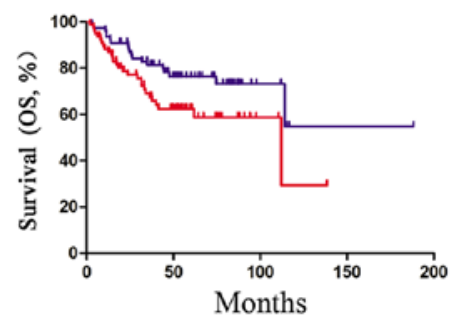

$\leftarrow$ ESRP1 expression $<$ Median - ESRP1 expression $>$ Median Log-rank $\mathrm{P}=0.0333$

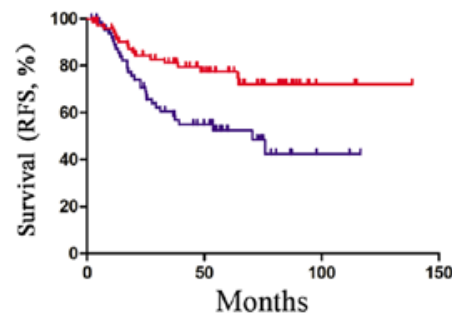

+ APOL2 expression < Median APOL2 expression $>$ Median Log-rank $\mathrm{P}=0.0035$

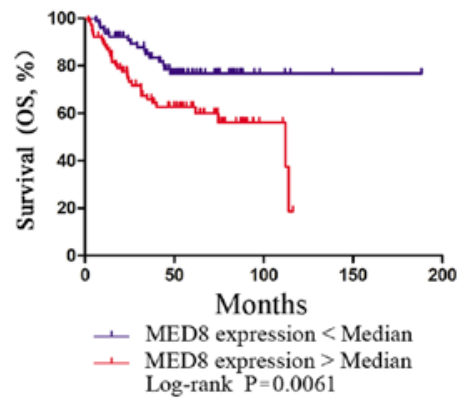

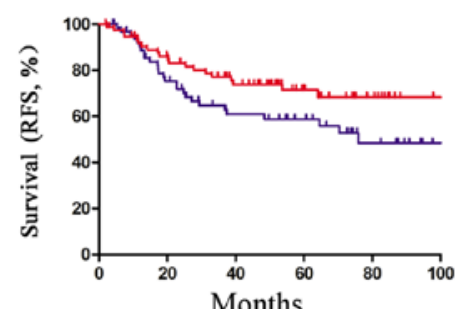

+ LDLRAD2 expression < Median + LDLRAD2 expression < Median
+ LDLRAD2 expression > Median Log-rank $\mathrm{P}=0.068$

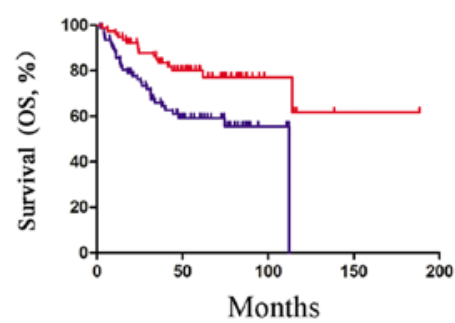

- EFCAB6 expression < Median - EFCAB6 expression > Median Log-rank $P=0.0027$

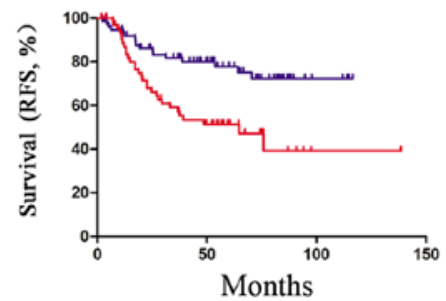

- ESRP1 expression $<$ Median - ESRP1 expression $>$ Median Log-rank $\mathrm{P}=0.0012$

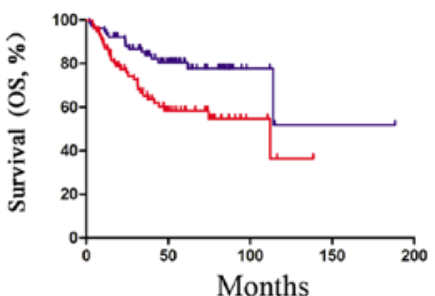

- DERL1 expression $<$ Median

- DERL1 expression $>$ Median Log-rank $\mathrm{P}=0.0052$

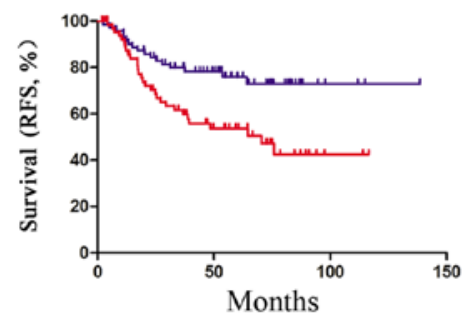

- MED8 expression < Median MED8 expression $>$ Median Log-rank $\mathrm{P}=0.0043$

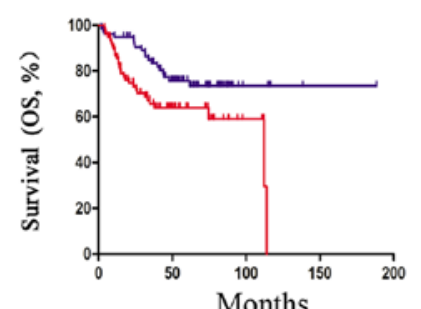

- DHCR24 expression < Median

DHCR24 expression $>$ Median Log-rank $\mathrm{P}=0.0122$

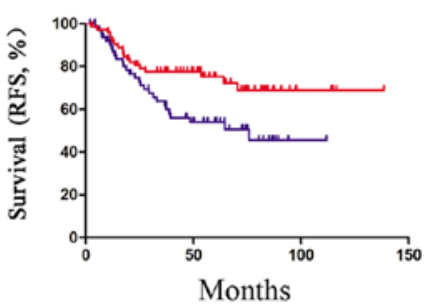

- EFCAB6 expression < Median - EFCAB6 expression > Median Log-rank $P=0.0201$

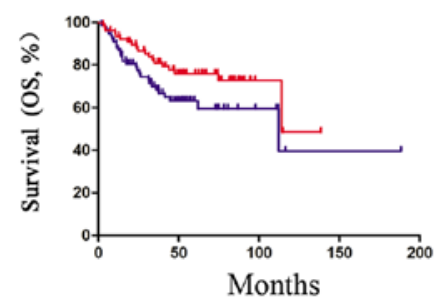

- APOL 2 expression < Median - APOL2 expression > Median Log-rank $\mathrm{P}=\mathbf{0 . 0 6 5 3}$

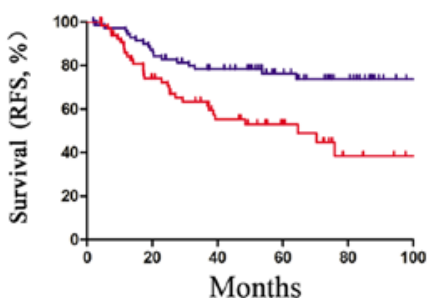

- - DERL1 expression < Median - DERL1 expression $>$ Median Log-rank $\mathrm{P}=0.0013$

Figure 3. Kaplan-Meier survival curves presenting the effects of expression of the 11 genes on the overall survival and relapse-free survival in patients with EEA in TCGA cohort. OS, overall survival; RFS, relapse-free survival; EEA, endometrioid endometrial adenocarcinoma; TCGA, The Cancer Genome Atlas; LDLRAD2, low density lipoprotein receptor class A domain-containing 2; DHCRAD2, 24-dehydrocholesterol reductase; EFCAB6, EF-hand calcium-binding domain-containing protein 6; ESRP1, epithelial-splicing-regulatory-protein 1; APOL2, apolipoprotein L2; DERL1, derlin-1; MED8, mediator complex subunit 8.

and traditional features serve important roles in EEA prognosis. Thus, a RF-combined model-3 for EEA prognosis was established by combining ' 11 genes and grade'. In the training set, the OOB error of RF model-3 established by ' 11 genes 
Table I. Clinicopathological characteristics of patients with EEA in TCGA and PKUPH cohorts.

\begin{tabular}{|c|c|c|c|c|c|c|c|c|}
\hline \multirow[b]{2}{*}{ Variable } & \multicolumn{3}{|c|}{ TCGA cohort } & \multirow[b]{2}{*}{ P-value } & \multicolumn{3}{|c|}{ PKUPH cohort } & \multirow[b]{2}{*}{ P-value } \\
\hline & $\begin{array}{l}\text { Overall } \\
(n=154)\end{array}$ & $\begin{array}{c}P E \\
(n=64)\end{array}$ & $\begin{array}{c}\text { R/D-PE } \\
(n=90)\end{array}$ & & $\begin{array}{l}\text { Overall } \\
(n=21)\end{array}$ & $\begin{array}{c}P E \\
(n=13)\end{array}$ & $\begin{array}{c}\text { R/D-PE } \\
(n=8)\end{array}$ & \\
\hline Age, years & & & & 0.09 & & & & 0.631 \\
\hline Median (range) & $64(35-90)$ & $62(35-89)$ & $65(35-90)$ & & $55(31-75)$ & $51(41-75)$ & $56(31-63)$ & \\
\hline$<60, \mathrm{n}$ & 55 & 28 & 27 & & 13 & 10 & 5 & \\
\hline$\geq 60, \mathrm{n}$ & 99 & 36 & 63 & & 8 & 3 & 3 & \\
\hline Grade, $\mathrm{n}$ & & & & 0.005 & & & & 0.930 \\
\hline $1-2$ & 80 & 42 & 38 & & 18 & 11 & 7 & \\
\hline 3 & 74 & 22 & 52 & & 3 & 2 & 1 & \\
\hline FIGO, n & & & & 0.005 & & & & 0.203 \\
\hline $\mathrm{I}$ & 105 & 52 & 53 & & 12 & 9 & 3 & \\
\hline II-IV & 49 & 12 & 37 & & 9 & 4 & 5 & \\
\hline Menopause status, $\mathrm{n}$ & & & & 0.789 & & & & 0.131 \\
\hline Premenopausal & 12 & 4 & 8 & & 4 & 4 & 0 & \\
\hline Postmenopausal & 129 & 54 & 75 & & 17 & 9 & 8 & \\
\hline Unknown & 13 & 6 & 7 & & 0 & 0 & 0 & \\
\hline ER status, $\mathrm{n}$ & & & & & & & & 0.133 \\
\hline Positive & & NA & & & 19 & 13 & 6 & \\
\hline Negative & & & & & 2 & 0 & 2 & \\
\hline PR status, $n$ & & & & & & & & 0.381 \\
\hline Positive & & NA & & & 20 & 13 & 7 & \\
\hline Negative & & & & & 1 & 0 & 1 & \\
\hline Lymph node status, $\mathrm{n}$ & & & & 0.008 & & & & 0.716 \\
\hline Positive & 22 & 4 & 18 & & 2 & 1 & 1 & \\
\hline Negative & 46 & 25 & 21 & & 19 & 12 & 7 & \\
\hline Unknown & 86 & 35 & 51 & & 0 & 0 & 0 & \\
\hline Adjuvant radiotherapy, $\mathrm{n}$ & & & & $<0.001$ & & & & 0.67 \\
\hline Yes & 41 & 0 & 41 & & 11 & 6 & 5 & \\
\hline No & 110 & 64 & 46 & & 9 & 6 & 3 & \\
\hline Unknown & 3 & 0 & 3 & & 1 & 1 & 0 & \\
\hline Adjuvant chemotherapy, $n$ & & & & & & & & 0.599 \\
\hline Yes & & NA & & & 13 & 7 & 6 & \\
\hline No & & & & & 6 & 4 & 2 & \\
\hline Unknown & & & & & 2 & 2 & 0 & \\
\hline BMI, n & & & & $<0.001$ & & & & 0.659 \\
\hline$<28$ & 40 & 40 & 0 & & 10 & 7 & 3 & \\
\hline$\geq 28$ & 108 & 24 & 84 & & 11 & 6 & 5 & \\
\hline Unknown & 6 & 0 & 6 & & 0 & 0 & 0 & \\
\hline
\end{tabular}

EEA, endometrioid endometrial adenocarcinoma; TCGA, The Cancer Genome Atlas; PKUPH, Peking University People's Hospital; PE, primary EEA samples; R/D-PE, relapsed or deceased primary EEA samples; FIGO, International Federation of Gynecology and Obstetrics; BMI, body mass index.

and grade' was 0.15 . When RF model-3 was used to validate the 21 EEA samples from the PKUPH cohort, its classification accuracy was $80.95 \%$ (Fig. 4B). The classification accuracy of the RF model established by '11 genes, grade and stage' was $80.95 \%$ (Fig. 4C), further proving that grade alone had equal classification ability compared with 'grade combined with FIGO stage'.

\section{Discussion}

Although the prognosis of EEA is good, extensive heterogeneity can expose patients to recurrent disease and poor prognosis $(3,4)$. Treatments for EEA have become more complicated, as the histological classification, adjuvant therapies, indications and modalities for lymphadenectomy, and 
Table II. Genes selected by Random Forest feature selection that may contribute to the prognosis of endometrial adenocarcinoma.

\begin{tabular}{lcl}
\hline Gene & Chromosome no. & \\
\hline LDLRAD2 & 1 & Low density lipoprotein receptor class A domain containing 2 \\
DHCR24 & 1 & 24-dehydrocholesterol reductase \\
EFCAB6 & 22 & EF-hand calcium-binding domain 6 \\
ESRP1 & 8 & Epithelial splicing regulatory protein 1 \\
APOL2 & 22 & Apolipoprotein L2 \\
DERL1 & 8 & Derlin 1 \\
MED8 & 1 & Mediator complex subunit 8 \\
PGAP3 & $\mathrm{c}$ & Post-GPI attachment to proteins 3 \\
ELAVL4 & 1 & Embryonic lethal abnormal visual system-like neuron-specific RNA binding protein 4 \\
TMEM27 & $\mathrm{X}$ & Transmembrane protein 27 \\
ATF7IP2 & 16 & Activating transcription factor 7 interacting protein 2 \\
\hline
\end{tabular}
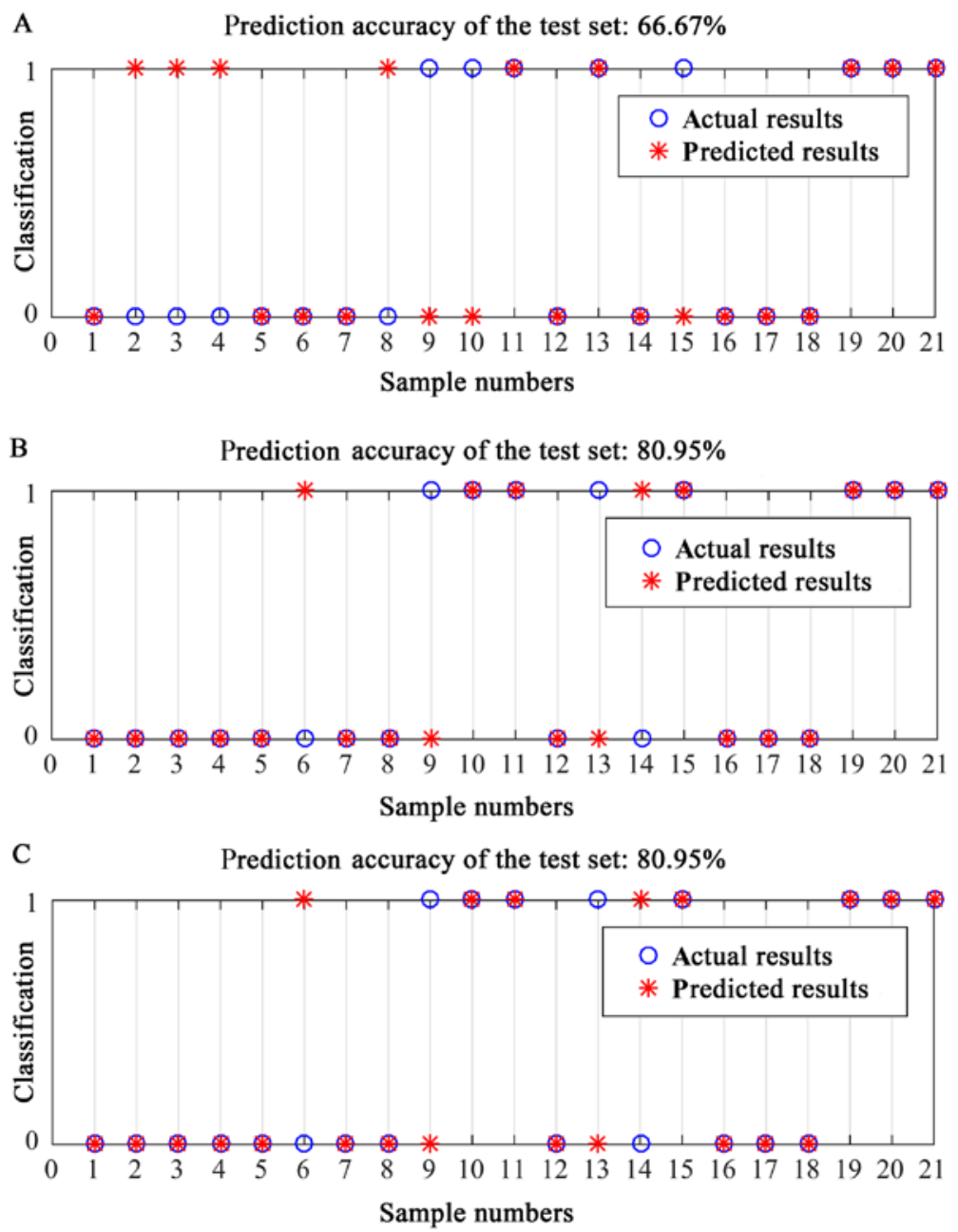

Figure 4. Prediction accuracy of using RF models for predicting endometrioid endometrial adenocarcinoma prognosis. (A) RF model established using grade, (B) RF model established using 11 genes and grade, (C) RF model established using 11 genes, grade and stage. RF, random forest.

the classifications used to predict relapse risk factors have all changed (21). Traditional clinical criteria are not enough to predict EEA prognosis accurately, although studies have demonstrated that a number of clinical factors, including tumor grade, age, comorbidities, tumor diameter, American Society of Anesthesiologists score (22), lymphovascular space involvement and postoperative complications at 30 days, serve important roles in the prognosis of endometrial cancer (23-25). For the limits of conventional traditional methods used for histological classification of endometrial cancer subtypes, Barlin et al suggested a combination of molecular and conventional characteristics as classifications for better appraisal of 
prognostic and predictive factors (26). Combining traditional clinical factors and molecular biological characteristics for the prognosis of EEA is important.

Machine-learning methods $(27,28)$ can provide increased prediction accuracy and can account for complex interactions among predictors. In addition, machine-learning approaches tend to be more suitable than traditional statistical methods for certain situations, such as cancer prognostic prediction, which involves a certain number of potential predictors (28). In machine learning, traditional classifiers are usually desired for prediction accuracy and easily fit in with clinical norms, whereas RF stands out for its own inherent characteristics, which include a better generalization performance and excellent classification results $(10,29)$. RF has also been demonstrated to be highly suitable for reducing the dimensionality of the data (29); it has been successfully used in numerous scientific realms, such as evaluating cancer-associated cognitive impairment, disease prediction, genetics, proteomics and informatics (29-31), but currently has no application in the prediction of EEA prognosis. Not only are RF good classifiers, but they are also increasingly used as feature-selection methods. In the present study, the VSURF method was used to identify informative factors that were relevant to the prognosis of EEA. The selected factors were then used to design a good RF predictive model.

In the present study, grade and 11 genes were selected for the establishment of an RF model. The selected 11 genes were involved in a number of important biological processes and potentially affect the prognosis of EC. LDLRAD2 is an integral component of the cell membrane. The present study indicated that LDLRAD2 was associated with the prognosis of EEA, but the definite biological significance of LDLRAD2 remains to be investigated. In addition, DHCR24 serves important roles in anti-apoptosis, cell cycle arrest, the negative regulation of cell death and the regulation of caspase activity; these biological processes are associated with poor prognosis (32). Dai et al (33) identified that insulin-induced cholesterol synthetase DHCR24 aggravates the invasion of cancer and the resistance to progesterone in endometrial carcinoma. A previous study also demonstrated that DHCR24 is able to predict poor clinicopathological features of patients with bladder cancer, and that its expression may promote bladder cancer cell proliferation via several oncogenesis-associated biological processes (for example, via estrogen response, heme metabolism, the p53 pathway, cholesterol homeostasis, mammalian target of rapamycin complex 1 signaling, peroxisomes, xenobiotic metabolism, glycolysis and protein secretion) (34). EFCAB6 and MED8 genes serve important roles in the transcription of genes, including certain prognosis-associated genes. ESRP1 and embryonic lethal abnormal visual system-like neuron-specific RNA-binding protein 4 (ELAVL4) participates in RNA processing, mRNA processing and mRNA metabolic processing. Li et al (35) demonstrated that ESRP1 inhibited the invasion and metastasis of lung adenocarcinoma, and served a role in regulating proteins involved in the epithelial-to-mesenchymal transition. ESRP1 was associated with prognosis in epithelial ovarian cancer (36) and human colorectal cancer (37). Expression of the ELAVL4 gene was demonstrated to be a diagnostic and prognostic marker of bone marrow lesions in patients with neuroblastoma and male patients with meningioma $(38,39)$. APOL2 serves important roles in the acute inflammatory response, lipid transport, the steroid metabolic process and the cholesterol metabolic process. APOL2 was found to be overexpressed in ovarian/peritoneal carcinoma and may provide a molecular basis for therapeutic target discovery (40). DERL1 participated in the endoplasmic reticulum (ER)-nuclear signaling pathway, the ER-associated protein catabolic process and the ER to cytosol process. The results of a previous study have indicated that the expression of DERL1 distinguishes malignant from benign canine mammary tumors (41). Post-glycosylphosphatidylinositol attachment to proteins 3 (PGAP3) is involved in protein amino acid lipidation, the glycerophospholipid metabolic process, the lipid biosynthetic process, the lipoprotein metabolic process and the lipoprotein biosynthetic process. Previous studies demonstrated that lipid metabolism disorders serve an important role in endometrial cancer $(42,43)$. PGAP3 may affect the prognosis of EEA by regulating the lipid metabolism process. Transmembrane protein 27 (TMEM27) serve important roles in proteolysis. Javorhazy et al (44) demonstrated that a lack of TMEM27 expression in conventional renal cell carcinoma defines a group of patients as at a high risk of cancer-associated mortality.

The use of the RF model for the prediction of EEA prognosis when deciding whether to recommend adjuvant therapies is of great importance, particularly for patients with FIGO stage I disease. Those who have a low risk of relapse according to traditional clinicopathological risk factors may not have to receive postoperative adjuvant chemoradiotherapy. The results from previous studies have indicated that a large proportion of patients with EEA, who were at a low risk of relapse according to the traditional criteria and had not received postoperative adjuvant chemoradiotherapy, eventually relapsed or deceased $(5,6)$. The RF prediction model derived on the basis of clinical features and gene expression is promising for providing an individualized and more accurate prediction for patients with EEA. Combining the predictive results of the RF model and traditional criteria could also be used for better stratification of patients in clinical trials, as well as for providing more accurate counseling ideas for patients.

Two nomograms $(45,46)$ established by traditional characteristics for the predictive survival of EC have been produced, and their training accuracies were between 0.71 and 0.78 . The first nomogram consists of five simple clinical features, including FIGO stage, age at diagnosis, final histological grade, negative lymph nodes and histological subtype (45). The second nomogram was validated in randomly selected patients (46) and indicated that tumor grade, age and lymphovascular space involvement were highly predictive for all outcomes. The establishment of the two nomograms was based on Cox regression analyses. Previous studies have demonstrated that machine-learning approaches appear to be more suitable than traditional statistical methods for some situations, such as the prediction of cancer prognosis, which involves a certain number of potential predictors $(10,11)$; thus, it may be more suitable to build such nomograms with machine-learning methods such as RF. In addition, biological characteristics and clinical features were particularly important in the prognosis of EEA. Using a combination of molecular and conventional characteristics as 
classifications would provide a better appraisal of prognostic and predictive factors, and the combination of traditional clinical factors and molecular biological characteristics is very important for the prognosis of EEA.

The classification accuracy of the RF prediction model combined with traditional clinicopathological features and gene expression was markedly higher than that of the RF models that were based on the traditional clinicopathological features or gene expression alone, indicating that traditional clinicopathological features and gene expression were important factors for the prognosis of EEA. The inclusion of numerous patients and prognosis-associated clinical features in the establishment of the RF prediction models is vital, and unfortunately, the number of samples in the present study was limited. In future research, more clinical samples and more clinical features will be collected for RF model establishment. The RF prediction model presented within the present study could provide a more individualized and accurate estimation of relapse and/or mortality for patients diagnosed with EEA following primary therapy. The RF model could also be used for better stratification of patients in clinical trials and for providing more accurate counseling ideas for patients.

To the best of our knowledge, the present study is the first to establish an EEA predictive model that combines genes and traditional features using RF. The RF model derived on the basis of the ' 11 genes and grade' achieved better predictive performances than RF models established by either the 11 genes or grade alone, indicating that the RF model derived on the basis of the 'genes and clinical features' had a stronger predictive ability for the prognosis of EEA.

\section{Acknowledgements}

Not applicable.

\section{Funding}

The present study was supported by the National Natural Science Foundation of China (grant nos. 81502237, 81272869 and 81672571), the National Key Technology Research and Development Program of the Ministry of Science and Technology of China (grant no. 2015BAI13B06), and the Basic research project of Peking University (grant no. BMU2018JC005).

\section{Availability of data and materials}

The datasets used and/or analyzed during the present study are available from the corresponding authors upon reasonable request.

\section{Authors' contributions}

FY and XS wrote the manuscript, collected the clinical information and performed the statistical analyses. LZ, XL, YC, JZ, $\mathrm{XH}$ and JL designed the study and revised the manuscript. SL analyzed the data. JW conceived and supervised the study and approved the final manuscript. All authors read and approved the manuscript and agree to be accountable for all aspects of the research to ensure that the accuracy or integrity of any part of the work is appropriately investigated and resolved.

\section{Ethics approval and consent to participate}

The present study was approved by the Ethics Committee of Peking University People's Hospital (Beijing, China). All participating patients received and provided written informed consent prior to joining the study.

\section{Patient consent for publication}

Not applicable.

\section{Competing interests}

The authors declare that they have no competing interests.

\section{References}

1. Albertini AF, Devouassoux-Shisheboran M and Genestie C: Pathology of endometrioid carcinoma. Bull Cancer 99: 7-12, 2012.

2. Piulats JM, Guerra E, Gil-Martin M, Roman-Canal B, Gatius S, Sanz-Pamplona R, Velasco A, Vidal A and Matias-Guiu X: Molecular approaches for classifying endometrial carcinoma. Gynecol Oncol 145: 200-207, 2017.

3. Bendifallah S, Ouldamer L, Lavoue V, Canlorbe G, Raimond E, Coutant C, Graesslin O, Touboul C, Collinet P, Darai E, et al: Patterns of recurrence and outcomes in surgically treated women with endometrial cancer according to ESMO-ESGO-ESTRO consensus conference risk groups: Results from the FRANCOGYN study group. Gynecol Oncol 144: 107-112, 2017.

4. Cancer Genome Atlas Research Network, Kandoth C, Schultz N, Cherniack AD, Akbani R, Liu Y, Shen H, Robertson AG, Pashtan I, Shen R, et al: Integrated genomic characterization of endometrial carcinoma. Nature 497: 67-73, 2013.

5. Marnitz S and Kohler C: Current therapy of patients with endometrial carcinoma. A critical review. Strahlenther Onkol 188: 12-20, 2012.

6. Wright JD, Barrena Medel NI, Sehouli J, Fujiwara K and Herzog TJ: Contemporary management of endometrial cancer. Lancet 379: 1352-1360, 2012.

7. Perou CM, Sorlie T, Eisen MB, van de Rijn M, Jeffrey SS, Rees CA, Pollack JR, Ross DT, Johnsen H, Akslen LA, et al: Molecular portraits of human breast tumours. Nature 406: 747-752, 2000

8. Yu KH, Zhang C, Berry GJ, Altman RB, Re C, Rubin DL and Snyder M: Predicting non-small cell lung cancer prognosis by fully automated microscopic pathology image features. Nat Commun 7: 12474, 2016.

9. Kleppe A, Albregtsen F, Vlatkovic L, Pradhan M, Nielsen B, Hveem TS, Askautrud HA, Kristensen GB, Nesbakken A, Trovik J, et al: Chromatin organisation and cancer prognosis: A pan-cancer study. Lancet Oncol 19: 356-369, 2018

10. Fernandez-Delgado M, Cernadas E, Barro S and Amorim D: Do we need hundreds of classifiers to solve real world classification problems? J Mach Learn Res 15: 3133-3181, 2014.

11. Diaz-Uriarte R and Alvarez de Andres S: Gene selection and classification of microarray data using Random Forest. BMC Bioinformatics 7: 3, 2006.

12. Moorthy K and Mohamad MS: Random Forest for gene selection and microarray data classification. Bioinformation 7: 142-146, 2011.

13. Eshaghi A, Wottschel V, Cortese R, Calabrese M, Sahraian MA, Thompson AJ, Alexander DC and Ciccarelli O: Gray matter MRI differentiates neuromyelitis optica from multiple sclerosis using Random Forest. Neurology 87: 2463-2470, 2016.

14. Llorens-Rico V, Lluch-Senar M and Serrano L: Distinguishing between productive and abortive promoters using a Random Forest classifier in Mycoplasma pneumoniae. Nucleic Acids Res 43: 3442-3453, 2015.

15. Cadenas JM, Garrido MC and Martinez R: Feature subset selection Filter-Wrapper based on low quality data. Expert Syst Appl 40: 6241-6252, 2013.

16. Diaz-Uriarte R: GeneSrF and varSelRF: A web-based tool and $\mathrm{R}$ package for gene selection and classification using Random Forest. BMC Bioinformatics 8: 328, 2007. 
17. Kwon SG, Hwang JH, Park DH, Kim TW, Kang DG, Kang KH, Kim IS, Park HC, Na CS, Ha J and Kim CW: Associated with litter size in ber identification of differentially expressed genes kshire pig placenta. PLoS One 11: e0153311, 2016.

18. Pecorelli S: Revised FIGO staging for carcinoma of the vulva, cervix, and endometrium. Int J Gynecol Obstet 105: 103-104, 2009.

19. Shepherd JH: Revised FIGO staging for gynaecological cancer. Br J Obstet Gynaecol 96: 889-892, 1989.

20. Ouldamer L, Bendifallah S, Body G, Touboul C, Graesslin O, Raimond E, Collinet P, Coutant C, Lavoue V, Leveque J, et al: Predicting poor prognosis recurrence in women with endometrial cancer: A nomogram developed by the FRANCOGYN study group. Br J Cancer 115: 1296-1303, 2016.

21. Morice P, Leary A, Creutzberg C, Abu-Rustum N and Darai E: Endometrial cancer. Lancet 387: 1094-1108, 2016.

22. Moreno RP,Pearse R and Rhodes A; European Surgical Outcomes Study (EuSOS) Group of the European Society of Intensive Care Medicine and European Society of Anaesthesiology Trials Groups: American society of anesthesiologists score: Still useful after 60 years? Results of the EuSOS study. Rev Bras Ter Intensiva 27: 105-112, 2015 (In English, Portuguese).

23. AlHilli MM, Mariani A, Bakkum-Gamez JN, Dowdy SC, Weaver AL, Peethambaram PP, Keeney GL, Cliby WA and Podratz KC: Risk-scoring models for individualized prediction of overall survival in low-grade and high-grade endometrial cancer. Gynecol Oncol 133: 485-493, 2014.

24. AlHilli MM, Podratz KC, Dowdy SC, Bakkum-Gamez JN, Weaver AL, McGree ME, Keeney GL, Cliby WA and Mariani A: Risk-scoring system for the individualized prediction of lymphatic dissemination in patients with endometrioid endometrial cancer. Gynecol Oncol 131: 103-108, 2013.

25. Creutzberg CL, van Putten WL, Koper PC, Lybeert ML, Jobsen JJ, Warlam-Rodenhuis CC, De Winter KA, Lutgens LC, van den Bergh AC, van de Steen-Banasik E, et al: Surgery and postoperative radiotherapy versus surgery alone for patients with stage- 1 endometrial carcinoma: Multicentre randomised trial. PORTEC study group. Post operative radiation therapy in endometrial carcinoma. Lancet 355: 1404-1411, 2000.

26. Barlin JN, Zhou Q, St Clair CM, Iasonos A, Soslow RA, Alektiar KM, Hensley ML, Leitao MM Jr, Barakat RR and Abu-Rustum NR: Classification and regression tree (CART) analysis of endometrial carcinoma: Seeing the forest for the trees. Gynecol Oncol 130: 452-456, 2013.

27. Jordan MI and Mitchell TM: Machine learning: Trends, perspectives, and prospects. Science 349: 255-260, 2015.

28. Burki TK: Predicting lung cancer prognosis using machine learning. Lancet Oncol 17: e421, 2016.

29. Sarica A, Cerasa A and Quattrone A: Random Forest algorithm for the classification of neuroimaging data in Alzheimer's disease: A systematic review. Front Aging Neurosci 9: 329, 2017.

30. Wang X, Lin P and Ho JW: Discovery of cell-type specific DNA motif grammar in cis-regulatory elements using Random Forest. BMC Genomics 19: 929, 2018

31. Taherzadeh G, Zhou Y, Liew AW and Yang Y: Structure-based prediction of protein-peptide binding regions using Random Forest. Bioinformatics 34: 477-484, 2018.

32. Dong W, Guan FF, Zhang X, Gao S, Liu N, Chen W, Zhang LF and Lu D: Dhcr24 activates the PI3K/Akt/HKII pathway and protects against dilated cardiomyopathy in mice. Animal Model Exp Med 1: 40-52, 2018.

33. Dai M, Zhu XL, Liu F, Xu QY, Ge QL, Jiang SH, Yang XM, Li J, Wang YH, Wu QK, et al: Cholesterol synthetase DHCR24 induced by insulin aggravates cancer invasion and progesterone resistance in endometrial carcinoma. Sci Rep 7: 41404, 2017.
34. Liu XP, Yin XH, Meng XY, Yan XH, Cao Y, Zeng XT and Wang XH: DHCR24 predicts poor clinicopathological features of patients with bladder cancer: A STROBE-compliant study. Medicine 97: e11830, 2018.

35. Li L, Qi L, Qu T, Liu C, Cao L, Huang Q, Song W, Yang L, Qi H, Wang Y, et al: Epithelial splicing regulatory protein 1 inhibits the invasion and metastasis of lung adenocarcinoma. Am J Pathol 188: 1882-1894, 2018

36. Chen L, Yao Y, Sun L, Zhou J, Miao M, Luo S, Deng G, Li J, Wang J and Tang J: Snail driving alternative splicing of CD44 by ESRP1 enhances invasion and migration in epithelial ovarian cancer. Cell Physiol Biochem 43: 2489-2504, 2017.

37. Fagoonee S, Picco G, Orso F, Arrigoni A, Longo DL, Forni M, Scarfo I, Cassenti A, Piva R, Cassoni P, et al: The RNA-binding protein ESRP1 promotes human colorectal cancer progression. Oncotarget 8: 10007-10024, 2017.

38. Dru1̆ AE, Tsaur GA, Popov AM, Tuponogov SN, Shorikov EV, Tsvirenko SV, Savel'ev LI and Fechina LG: The TH, ELAVL4 and GD2 gene expression as diagnostic markers of bone marrow lesions in patients with neuroblastoma. Vopr Onkol 58: 514-520, 2012 (In Russian).

39. Stawski R, Piaskowski S, Stoczynska-Fidelus E, Wozniak K, Bienkowski M, Zakrzewska M, Witusik-Perkowska M, Jaskolski DJ, Och W, Papierz W, et al: Reduced expression of ELAVL4 in male meningioma patients. Brain Tumor Pathol 30: 160-166, 2013.

40. Davidson B, Stavnes HT, Holth A, Chen X, Yang Y, Shih IM and Wang TL: Gene expression signatures differentiate ovarian/peritoneal serous carcinoma from breast carcinoma in effusions. J Cell Mol Med 15: 535-544, 2011.

41. Klopfleisch R, Klose P and Gruber AD: The combined expression pattern of BMP2, LTBP4, and DERL1 discriminates malignant from benign canine mammary tumors. Vet Pathol 47: 446-454, 2010.

42. Hirasawa A, Makita K, Akahane T, Yokota M, Yamagami W, Banno K, Susumu N and Aoki D: Hypertriglyceridemia is frequent in endometrial cancer survivors. Jpn J Clin Oncol 43: 1087-1092, 2013

43. Trousil S, Lee P, Pinato DJ, Ellis JK, Dina R, Aboagye EO, Keun HC and Sharma R: Alterations of choline phospholipid metabolism in endometrial cancer are caused by choline kinase alpha overexpression and a hyperactivated deacylation pathway. Cancer Res 74: 6867-6877, 2014.

44. Javorhazy A, Farkas N, Beothe T, Pusztai C, Szanto A and Kovacs G: Lack of TMEM27 expression is associated with postoperative progression of clinically localized conventional renal cell carcinoma. J Cancer Res Clin Oncol 142: 1947-1953, 2016.

45. Abu-Rustum NR, Zhou Q, Gomez JD, Alektiar KM, Hensley ML, Soslow RA, Levine DA, Chi DS, Barakat RR and Iasonos A: A nomogram for predicting overall survival of women with endometrial cancer following primary therapy: Toward improving individualized cancer care. Gynecol Oncol 116: 399-403, 2010.

46. Creutzberg CL, van Stiphout RG, Nout RA, Lutgens LC, Jurgenliemk-Schulz IM, Jobsen JJ, Smit VT and Lambin P: Nomograms for prediction of outcome with or without adjuvant radiation therapy for patients with endometrial cancer: A pooled analysis of PORTEC-1 and PORTEC-2 trials. Int J Radiat Oncol Biol Phys 91: 530-539, 2015.

(i) (3) This work is licensed under a Creative Commons Attribution-NonCommercial-NoDerivatives 4.0 International (CC BY-NC-ND 4.0) License. 